\title{
Cause analysis of vibration in shell-and-tube heat exchanger
}

\author{
Jia Wu ${ }^{1,2}$, Jiansheng $\mathrm{Hu}^{1,2}$, Bin $\mathrm{Du}^{1,2, *}$, Ping Tang ${ }^{1,2}$ and Jie Tang ${ }^{1,2}$ \\ ${ }^{1}$ Zhejiang Academy of Special Equipment Science, Hangzhou, China \\ ${ }^{2}$ Key Laboratory of Special Equipment Safety Testing Technology of Zhejiang Province, Hangzhou, China
}

\begin{abstract}
Heat exchanger is a universal process equipment, which is widely used in chemical industry, oil refining, thermal power and other industries. Shell-and-tube heat exchangers often fail prematurely due to flow-induced vibration. In this paper, aiming at the problem of severe vibration of shell-and-tube heat exchanger in an enterprise, the vibration acceleration sensors are used to measure the vibration of shell and tube side of heat exchanger. Through the analysis of the measurement results, it is found that when the flow rate on the shell side of the heat exchanger is greater than $50000 \mathrm{Nm}^{3} / \mathrm{h}$, the flow-induced vibration of the tube bundle occurs, and the vibration frequency is $57 \mathrm{~Hz}$, which is close to the natural frequency of the tube bundle. And the greater the flow rate on the shell side, the more intense the vibration. In order to reduce the vibration of heat exchanger, the damping measure of inserting baffles between tubes is adopted.
\end{abstract}

\section{Introduction}

Shell-and-tube heat exchanger is a widely used process equipment in petroleum, chemical, food and pharmaceutical industries. With the development of social economy and the expansion of industrial production scale, shell-and-tube heat exchangers tend to be large-scale. Moreover, due to the increase of heat exchanger size and tube bundle support spacing, the increase of fluid flow rate, unstable operating conditions and other factors, flow-induced vibration of heat exchanger tube bundle is often caused, resulting in local failure or even overall scrapping of heat exchanger, bring huge economic losses to the factories. There are three main reasons for tube bundle vibration induced by fluid in shell-and-tube heat exchanger, namely vortex shedding, fluid-elastic instability and turbulent buffeting [1].

When the fluid of the shell side flows through the tubes of the heat exchanger, an alternating Karman vortex street will be formed downstream of the tubes, resulting in a periodically varying exciting force, which is perpendicular to the fluid flow direction and leads to tube vibration. When the vortex shedding frequency is consistent with the natural frequency of the tube, violent vibration will occur. In the past decades, many scholars have been studying the vortex shedding phenomenon. Grotz and Rarnold [2] studied the vortex shedding phenomenon in shell-and-tube heat exchangers, proposed a set of design factors, and revealed the relationship between tube spacing ratio and vortex shedding frequency. Lienhard [3] found that for the tube bundle with vortex shedding, the Strouhal number is a function of the tube arrangement. Pettigrew and Taylor [4] formulated design guidelines for prevention of tube bundle failure caused by excessive flow-induced vibration in shell-and-tube heat exchangers. Khushnood and Nizam [5] analyzed the vibration response of tube bundle under two different flow conditions, and studied the influence of damping on tube vibration behavior. Ji et al. [6, 7] discussed the influence of fluid velocity and structural parameters of shell side on flow-induced vibration response of elastic tube bundle.

Fluid-elastic instability is also one of the main causes of heat exchanger tube failure. When the fluid flows through the tube bundle, due to the action of turbulence, some tubes in the tube bundle may move instantaneously, so as to change the flow field around them, destroy the force balance on adjacent tubes, make the tubes move and begin to vibrate. If the amplitude continues to increase, fluid-elastic instability will occur [8]. Roberts [9] found that the displacement mechanism of the tube can be explained as dynamic instability of the tube bundle in the cross flow, and experimentally determined that the alternating motion of the tube will change the wake pairing downstream of the tube bundle. Chen [10] summarized different instability models and stability diagrams to avoid fluid-elastic instability. Fricker [11] found that the clearance between the tube and the support is prone to fluid-elastic instability, so as to predict the vibration characteristics and impact behavior of the tube. Gordon and Lebret [12] analyzed the influence of clearance on fluid-elastic instability through experiments. According to the unsteady flow theory, Cai et al. [13] established the mathematical model of fluid-elastic instability of loosely supported tube in non-uniform cross flow, simulated and studied the characteristics of fluidelastic instability, and compared the analysis results with the published experimental data. Weaver and Parrondo [14] conducted experimental study on the fluid-elastic instability of tube bundle of multi span heat exchangers, and conservatively estimated the critical velocities of

\footnotetext{
* Corresponding author e-mail: wujiachn@126.com
} 
seven different configurations according to the semi empirical model and the observed critical instability model. Lin and $\mathrm{Yu}$ [15] discussed the problem of fluidelastic instability by taking multiple cylinders under cross flow as the research object. The results show that when the velocity is higher than the critical value, high amplitude fluid-elastic vibration will occur in multiple cylinders; Above the critical velocity, the cylinders begin to vibrate along the elliptical orbit. Bao et al. [16] theoretically deduced the fluid-elastic excitation on the shell side of the heat exchanger, and analyzed the vibration of the tube bundle at different positions by numerical simulation method.

When the fluid force is the only function of time, it will produce forced vibration, that is, turbulent buffeting. Turbulence is characterized by the random variation of flow pattern and fluid velocity around the tube $[17,18]$. De Paula et al. [19] discussed some characteristics of turbulent flow in triangular arranged tubes through hot wire measurement in aerodynamic channels and flow visualization in waterways. Ishihara [20] conducted experiments with actual heat exchangers, measured the vibration of a large number of tubes under different flow rates, and measured natural frequencies and the damping ratio of the tubes. The results show that the cause of tube vibration is the buffeting caused by upstream turbulence. These tubes are supported by many baffles with gaps, which makes the vibration system nonlinear. Goyder [21] pointed out that compared with fluid-elastic instability, the vibration caused by turbulent buffeting is very small. However, after many years of use, due to fretting wear, this relentless low-level vibration will lead to the thinning of the tube wall, which is unacceptable in high-integrity heat exchangers.

In this paper, aiming at the problem of severe vibration of shell-and-tube heat exchanger in an enterprise, the vibration acceleration sensors are used to measure the vibration of shell and tube side of heat exchanger. By analyzing the vibration characteristics, the cause of vibration of heat exchanger is found out, and the vibration reduction measure is put forward.

\section{Measuring method}

\subsection{Heat exchanger model}

Flue gas heat exchanger is used to heat the flue gas treated by the desulfurization system to raise the temperature before entering the chimney and improve the corrosion of the tail flue and chimney. The model of a flue gas heat exchanger system of an enterprise is shown in Figure 1. The type of the heat exchanger is shell-and-tube heat exchanger, which uses the capacity of induced draft fan to guide the flue gas.

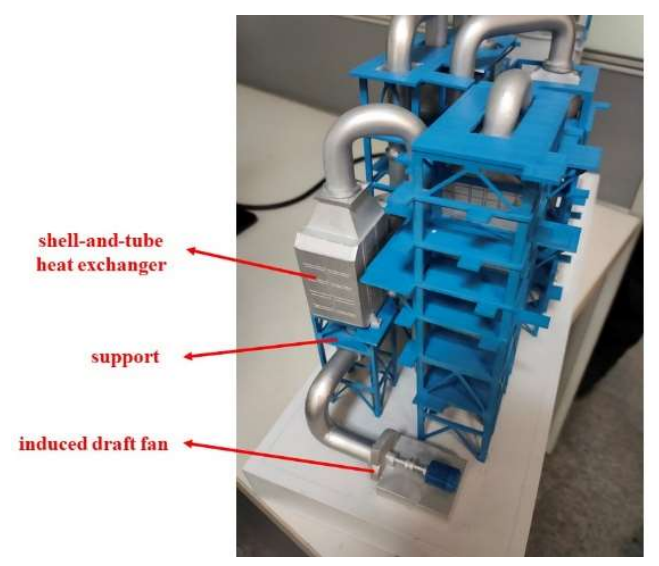

Figure 1. Model of flue gas heat exchanger system

In the shell-and-tube heat exchanger, the stroke of the fluid for heat exchange in the tube bundle is called tube side, and the stroke of the fluid for heat exchange outside the tube bundle is called shell side. The wall of the tube bundle is the heat transfer surface of cold and hot fluid. The tube side and shell side of shell-and-tube heat exchanger are shown in Figure 2, and the process parameters are listed in Table 1.

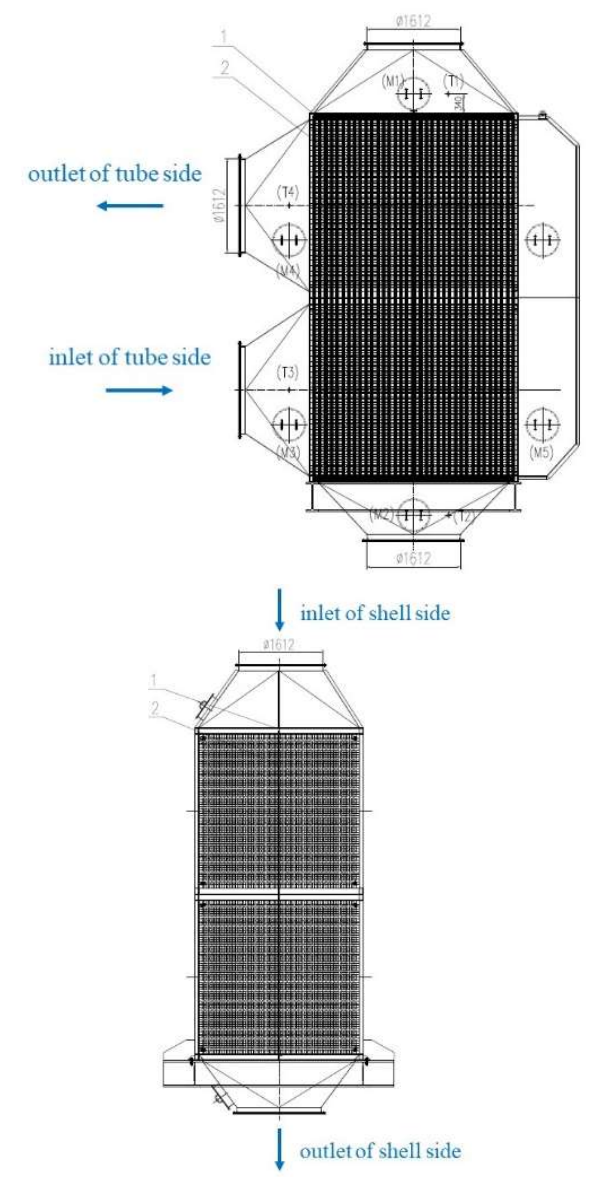

Figure 2. Tube side and shell side 
Table 1. Process parameters of shell-and-tube heat exchanger

\begin{tabular}{ccc}
\hline & Shell side & Tube side \\
\hline $\begin{array}{c}\text { Inlet / outlet } \\
\text { temperature }\end{array}$ & $180^{\circ} \mathrm{C} / 145^{\circ} \mathrm{C}$ & $115^{\circ} \mathrm{C} / 150^{\circ} \mathrm{C}$ \\
Pressure drop & $1100 \mathrm{~Pa}$ & $700 \mathrm{~Pa}$ \\
$\begin{array}{c}\text { Design flow rate } \\
\text { of flue gas }\end{array}$ & $72000 \mathrm{Nm}^{3} / \mathrm{h}$ & $72000 \mathrm{Nm}^{3} / \mathrm{h}$ \\
$\begin{array}{c}\text { Flue gas velocity } \\
\text { Thermal load }\end{array}$ & $6.4 \mathrm{~m} / \mathrm{s}$ & $12.4 \mathrm{~m} / \mathrm{s}$ \\
\hline
\end{tabular}

\subsection{Pulse excitation method}

The measurement of natural frequencies of tube side and shell side in shell-and-tube heat exchanger is carried out by pulse excitation method, and its principle is as follows:

The function $\delta$ represents an instantaneous pulse and if the force acting on the object is a unit impulse $\delta(t)$, then:

$$
\delta(t)=\left\{\begin{array}{ll}
\infty & t=0 \\
0 & t>0
\end{array} \text { and } \int_{-\infty}^{+\infty} \delta(t) d t=1\right.
$$

In a linear system, assuming that the input signal is $x(t)$, the mathematical operation of the system on the input signal is represented by the symbol $\mathrm{L}$, and the output of the system is $y(t)$, then:

$$
y(t)=L[x(t)]
$$

In order to obtain the frequency domain characteristics of the system, Fourier transform is performed on $x(t), y(t)$ and system impulse response $h(t)$, and the results are represented by symbols $X(f), Y(f)$ and $H(f)$, respectively, then:

$$
\begin{aligned}
Y(f) & =\int_{-\infty}^{+\infty} y(t) e^{-j 2 \pi f t} d t \\
& =\int_{-\infty}^{+\infty}\left[\int_{-\infty}^{\infty} x(\tau) h(t-\tau) d \tau\right] e^{-j 2 \pi f t} d t \\
& =\int_{-\infty}^{+\infty} x(\tau)\left[\int_{-\infty}^{\infty} h(t-\tau) e^{-j 2 \pi f(t-\tau)} d t\right] e^{-j 2 \pi f t} d \tau \\
& =\int_{-\infty}^{+\infty} x(\tau) H(f) e^{-j 2 \pi f \tau} d \tau \\
& =X(f) H(f)
\end{aligned}
$$

Because when the input signal $x(t)$ is equal to $\delta(t)$, its Fourier transform $X(f)$ is equal to 1 . It can be obtained from the above formula:

$$
H(f)=\frac{Y(f)}{X(f)}=\int_{-\infty}^{+\infty} y(t) e^{-j 2 \pi f t} d t
$$

This shows that if a unit impulse force is applied to the measured system, as long as the response of the system to the impulse force is measured and Fourier transformed, the frequency response function of the system can be obtained, which represents the dynamic characteristics of the system.

\section{Measurement results}

\subsection{Natural frequency measurement}

The natural frequency measurement is carried out by pulse excitation method. By analyzing the frequency spectrum of pulse response, the natural frequency of tube side and shell side can be obtained.

Figure 3 shows the vibration spectra of the tube side without excitation and excitation, and Figure 4 shows the vibration spectra of the shell side without excitation and excitation.

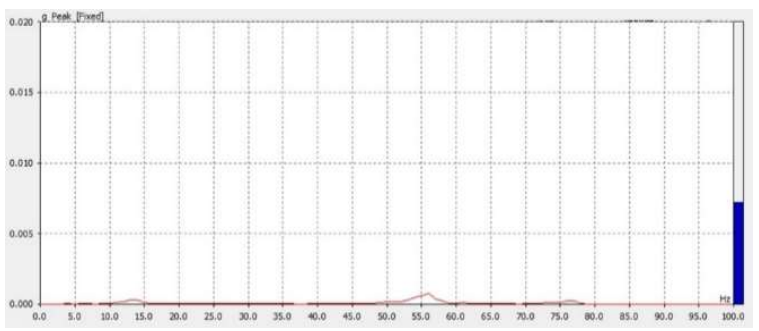

(a) without excitation

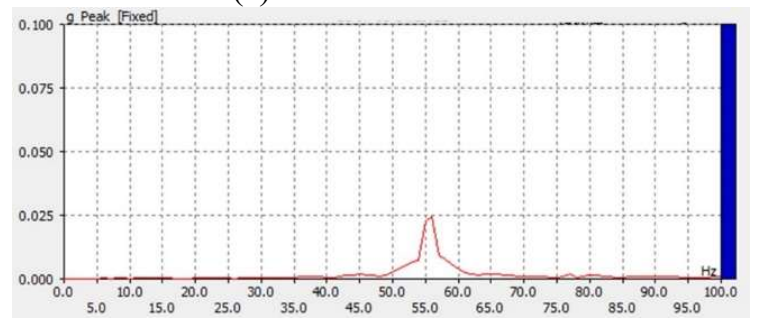

(b) under excitation

Figure 3. Vibration spectra of tube side without excitation and excitation

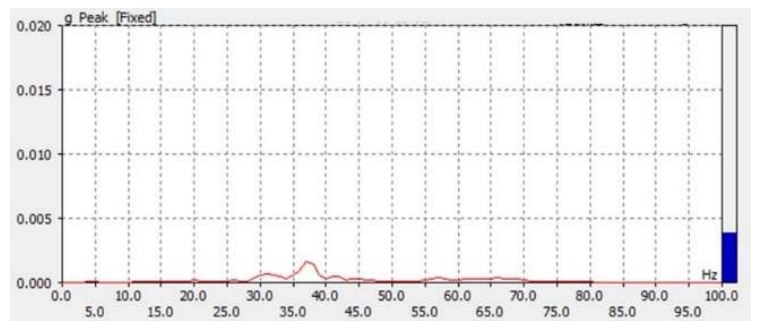

(a) without excitation

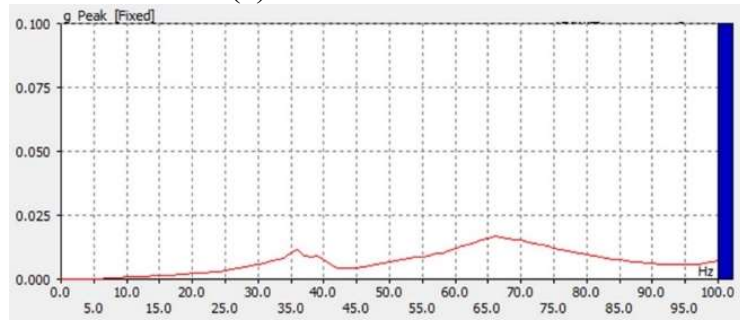

(b) under excitation

Figure 4. Vibration spectra of shell side without excitation and excitation

As can be seen from Figure 3, when not excited, the frequency of the tube side is mainly $56 \mathrm{~Hz}$, while when excited, the frequency still exists, but the amplitude increases sharply. This indicates that the natural frequency of the tube side is $56 \mathrm{~Hz}$. Similarly, it can be seen from Figure 4 that the natural frequencies of the shell side are $37 \mathrm{~Hz}$ and $66 \mathrm{~Hz}$.

\subsection{Comparison of vibration under different flow rates}

Figure 5 9 shows the vibration of shell-and-tube heat exchanger under five different flow rates. It can be seen from the figures that when the flow rate is $45000 \mathrm{Nm}^{3} / \mathrm{h}$ and $48000 \mathrm{Nm}^{3} / \mathrm{h}$, the vibration is small, and the main vibration frequency is about $37.5 \mathrm{~Hz}$, which is very close 
to the natural frequency of $37 \mathrm{~Hz}$ on the shell side. When the flow rate is greater than $50000 \mathrm{Nm}^{3} / \mathrm{h}$, the vibration increases significantly, and the main vibration frequency is about $57 \mathrm{~Hz}$, which is close to the natural frequency of the tube side. It shows that when the flow rate increases to a certain extent, flow-induced vibration occurs in the shell-and-tube heat exchanger.

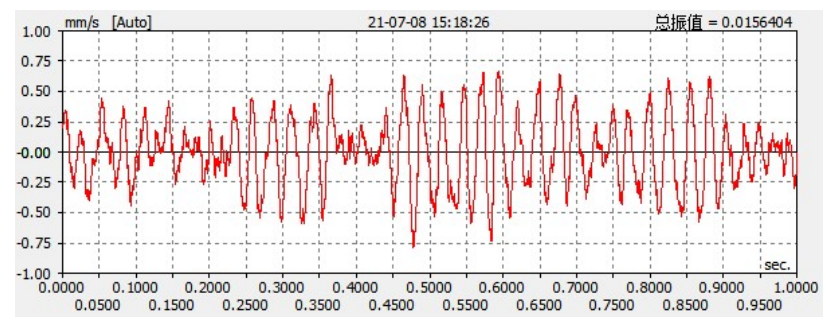

(a) vibration velocity waveform

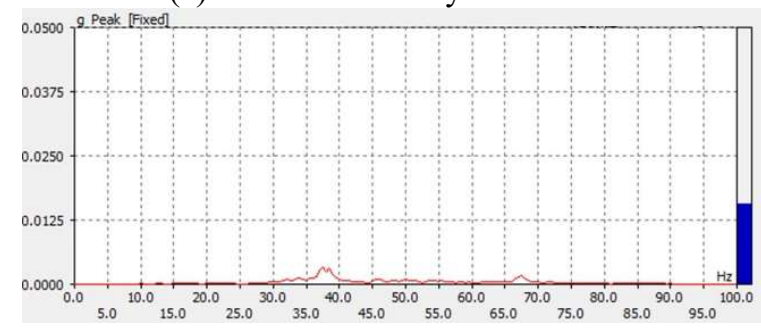

(b) frequency spectrum

Figure 5. Vibration when the flow rate is $45000 \mathrm{Nm}^{3} / \mathrm{h}$

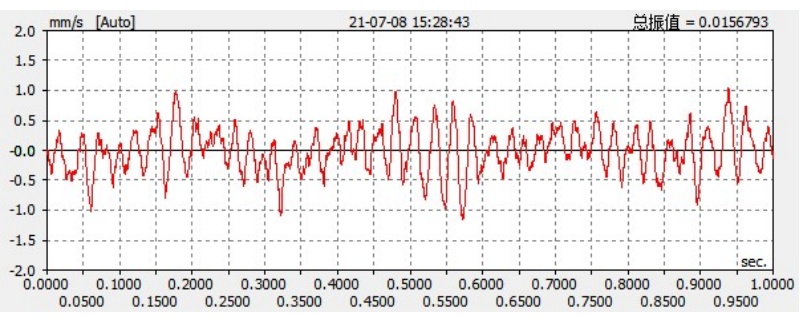

(a) vibration velocity waveform

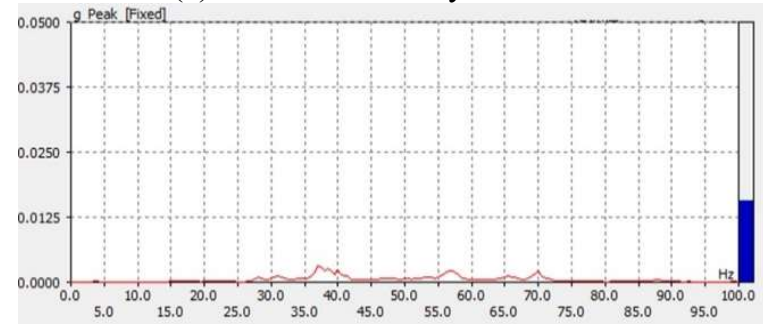

(b) frequency spectrum

Figure 6. Vibration when the flow rate is $48000 \mathrm{Nm}^{3} / \mathrm{h}$

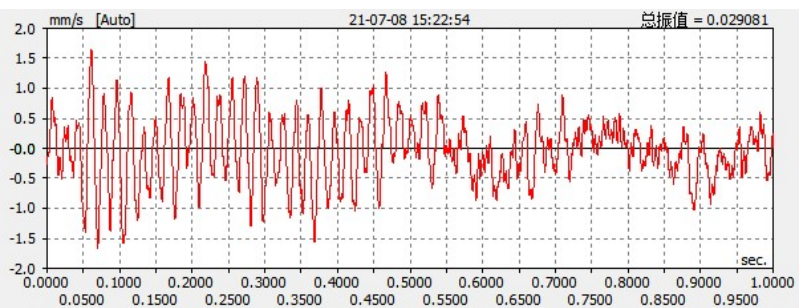

(a) vibration velocity waveform

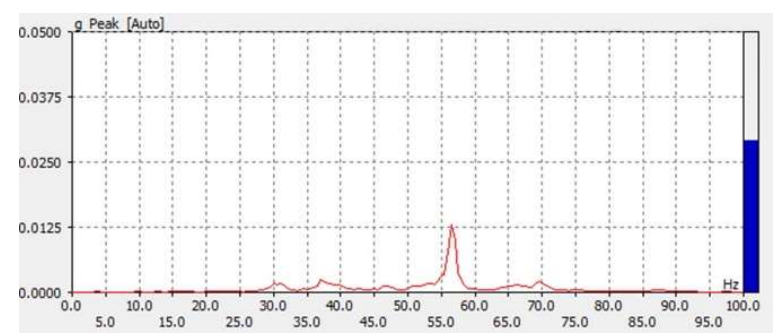

(b) frequency spectrum

Figure 7. Vibration when the flow rate is $50000 \mathrm{Nm}^{3} / \mathrm{h}$

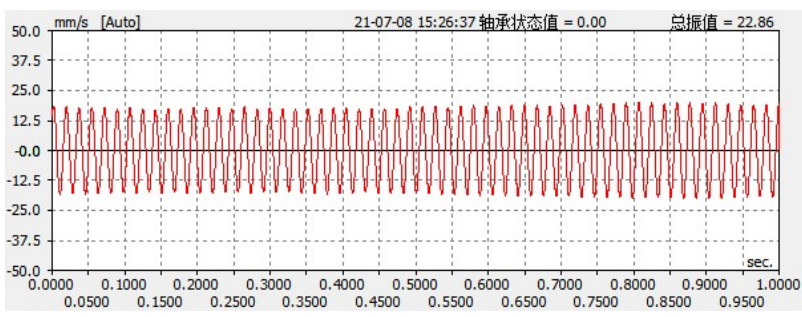

(a) vibration velocity waveform

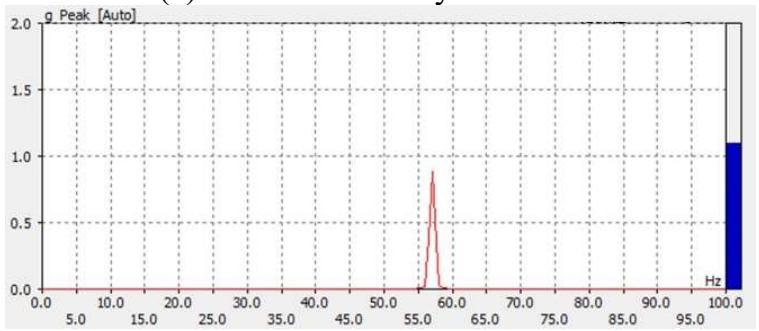

(b) frequency spectrum

Figure 8. Vibration when the flow rate is $51000 \mathrm{Nm}^{3} / \mathrm{h}$

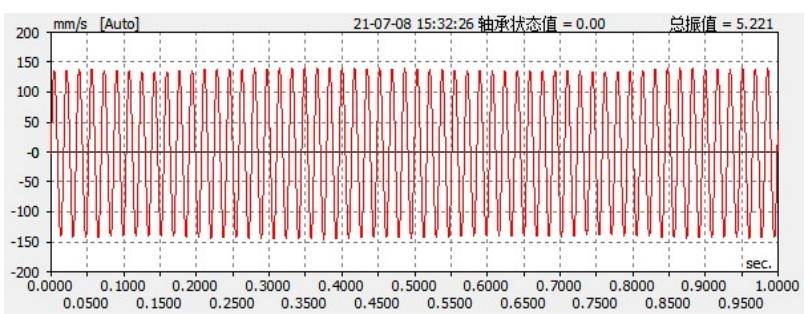

(a) vibration velocity waveform

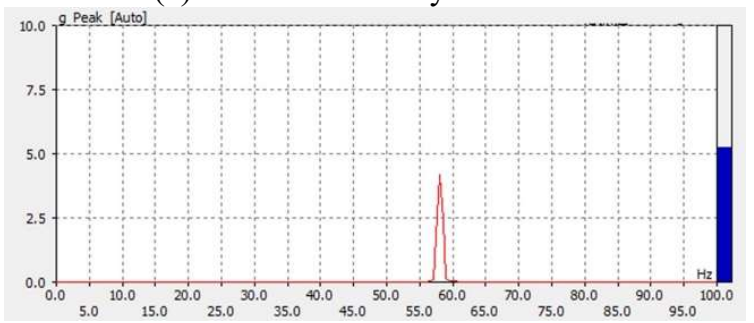

(b) frequency spectrum

Figure 9. Vibration when the flow rate is $55000 \mathrm{Nm}^{3} / \mathrm{h}$

\section{Vibration reduction measure}

The vibration of the tube in heat exchanger is inevitable. Slight vibration will not cause damage to the heat exchanger, but also strengthen heat transfer and reduce scaling. However, for strong vibration, necessary anti vibration measures shall be taken to slow down the vibration and avoid vibration damage of heat exchanger. According to the above analysis, the fundamental way to 
reduce vibration is to avoid the natural frequency of the tube as far as possible.

Because the natural frequency of the heat exchange tube is inversely proportional to the square of the supporting span of the tube bundle, reducing the span can improve the natural frequency of the heat exchange tube, which is the most economical and effective vibration reduction measure in engineering practice. In Figure 10, two baffles are inserted between the heat exchange tubes, which reduces the span, strengthens the tube bundle and changes the natural frequency of the heat exchange tube.

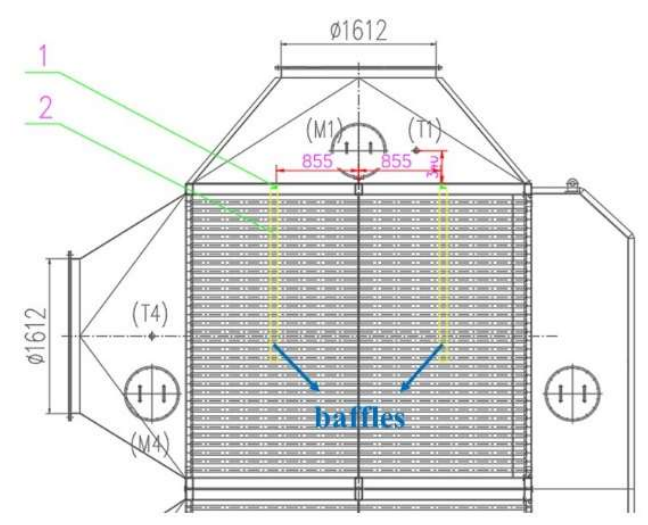

Figure 10. Baffles inserted between heat exchange tubes

\section{Conclusions}

(1) The vibration of the shell-and-tube heat exchanger is caused by flow-induced vibration of tube bundle. When the flow rate on the shell side of the heat exchanger is greater than $50000 \mathrm{Nm}^{3} / \mathrm{h}$, flow-induced vibration is generated, and the vibration frequency is $57 \mathrm{~Hz}$, which is close to the natural frequency of the tube bundle. And the greater the flow rate on the shell side, the greater the impact on the tube bundle and the more intense the vibration.

(2) In order to reduce the vibration of the heat exchanger, it is suggested to insert baffles between the heat exchange tubes, so as to change the natural frequency of the heat exchange tubes and avoid the frequency of exciting force.

\section{Acknowledgments}

This work was financially supported by the Natural Science Foundation of Zhejiang Province (NO. LQ21E050001) and the science and technology project of Zhejiang Provincial Administration for Market Regulation (NO. CY2022221).

\section{References}

1. Weaver, D. T., and Fitzpatrick, J. A. (1988). A review of cross-flow induced vibrations in heat exchanger tube arrays. Journal of fluids and structures, 2(1), 7393.
2. Grotz, B. J., and Rarnold, F. (1956). Flow-induced vibrations in Heat Exchangers. Stanford University, Department of Mechanical Engineering.

3. Lienhard, J. H. (1966). Synopsis of lift, drag, and vortex frequency data for rigid circular cylinders (Vol. 300). Pullman, WA: Technical Extension Service, Washington State University.

4. Pettigrew, M. J., and Taylor, C. E. (2003). Vibration analysis of shell-and-tube heat exchangers: an overview_Part 2: vibration response, fretting-wear, guidelines. Journal of Fluids and Structures, 18(5), 485-500.

5. Khushnood, S., and Nizam, L. A. (2017). Experimental study on cross-flow induced vibrations in heat exchanger tube bundle. China Ocean Engineering, 31(1), 91-97.

6. Ji, J., Ge, P., and Bi, W. (2016). Numerical analysis on shell-side flow-induced vibration and heat transfer characteristics of elastic tube bundle in heat exchanger. Applied Thermal Engineering, 107, 544551 .

7. Ji, J. D., Ge, P. Q., and Bi, W. B. (2018). Numerical analysis of shell-side flow-induced vibration of elastic tube bundle in heat exchanger. Journal of Hydrodynamics, 30(2), 249-257.

8. Price, S. J. (1995). A review of theoretical models for fluidelastic instability of cylinder arrays in cross-flow. Journal of Fluids and Structures, 9(5), 463-518.

9. Roberts, B. W. (1966). Low frequency, aeroelastic vibrations in a cascade of circular cylinders. Mechanical Science Monograph, (4).

10. Chen, S. S. (1984). Guidelines for the instability flow velocity of tube arrays in crossflow. Journal of Sound and Vibration, 93(3), 439-455.

11. Fricker, A. J. (1992). Numerical analysis of the fluidelastic vibration of a steam generator tube with loose supports. Journal of Fluids and Structures, 6(1), 85-107.

12. Godon, J. L., and Lebret, J. (1988). Influence of the tube-support plate clearance on flow-induced vibration in large condensers. In Proceedings of the International Symposium on Flow-Induced Vibration and Noise: Flow-Induced Vibration in Heat-Transfer Equipment, Chicago, IL (pp. 177-186).

13. Cai, Y., Chen, S. S., and Chandra, S. (1992). A theory for fluidelastic instability of tube-support-plateinactive modes. ASME. J. Pressure Vessel Technol, 114(2): 139-148.

14. Weaver, D. S., and Parrondo, J. (1991). Fluidelastic instability in multispan heat exchanger tube arrays. Journal of fluids and structures, 5(3), 323-338.

15. Lin, T. K., and Yu, M. H. (2005). An experimental study on the cross-flow vibration of a flexible cylinder in cylinder arrays. Experimental thermal and fluid science, 29(4), 523-536.

16. Bao, M., Wang, L., Li, W., and Gao, T. (2017). The Vibration Analysis of Tube Bundles Induced by Fluid Elastic Excitation in Shell Side of Heat Exchanger. In 
IOP Conference Series: Materials Science and Engineering (Vol. 239, No. 1, p. 012011). IOP Publishing.

17. Blevins, R. D. (1977). Flow-induced vibration. New York.

18. Polak, D. R., and Weaver, D. S. (1995). Vortex shedding in normal triangular tube arrays. Journal of Fluids and Structures, 9(1), 1-17.

19. De Paula, A. V., Endres, L. A. M., and Möller, S. V. (2012). Bistable features of the turbulent flow in tube banks of triangular arrangement. Nuclear engineering and design, 249, 379-387.

20. Ishihara, K. (2007). Flow Induced Vibration of Shell \& Tube Type Heat Exchanger (1st Report, Understanding of Phenomena). Journal of Environment and Engineering, 2(2), 293-302.

21. Goyder, H. G. D. (2002). Flow-induced vibration in heat exchangers. Chemical Engineering Research and Design, 80(3), 226-232. 